\title{
Passively Mode-Locked Fiber Laser Incorporating Adaptive Filtering and Dispersion Management
}

\author{
Xin Yang, Kamal Hammani, David J. Richardson, and Periklis Petropoulos \\ Optoelectronic Research Centre, University of Southampton, Highfield, Southampton, United Kingdom, SO17 1 BJ \\ xy1v07@orc.soton.ac.uk
}

\begin{abstract}
We present the operation of a passively mode-locked Erbium (Er)-doped fiber ring laser that incorporates a fully programmable filter, allowing control of the cavity net dispersion. OCIS codes: (140.3510) Lasers, fiber; (190.4370) Nonlinear optics, fibers
\end{abstract}

\section{Introduction}

The exact dispersion profile of a pulsed fiber laser cavity is a fundamental design consideration, since it critically affects the characteristics of the pulses generated in the cavity and determines the pulse formation mechanism. In the past, several methods have been employed to achieve flexible control of intra-cavity dispersion, including grating pairs, prisms or simply physical changing the length of the fiber in the cavity $[1,2]$. All these techniques however, require manual tuning of some physical parameters of the cavity. Recently, fully programmable phase and amplitude filters [3] have become available commercially and are already extensively used in telecommunications applications [4]. The use of such filters, when placed inside a laser cavity, has the potential to allow the operation of lasers that exhibit pulse characteristics that can be controlled purely through software control [5].

In the work that we present in this paper, we demonstrate the operation of a passively mode-locked fiber laser that incorporated a programmable filter within the ring cavity. The net dispersion (ND) of the cavity was controlled by applying different group velocity dispersion (GVD) profiles on the transfer function of the programmable filter. This allowed the laser pulse characteristics to be controlled without altering the pump power or applying any physical changes in the layout of the laser cavity.

\section{Laser Configuration}

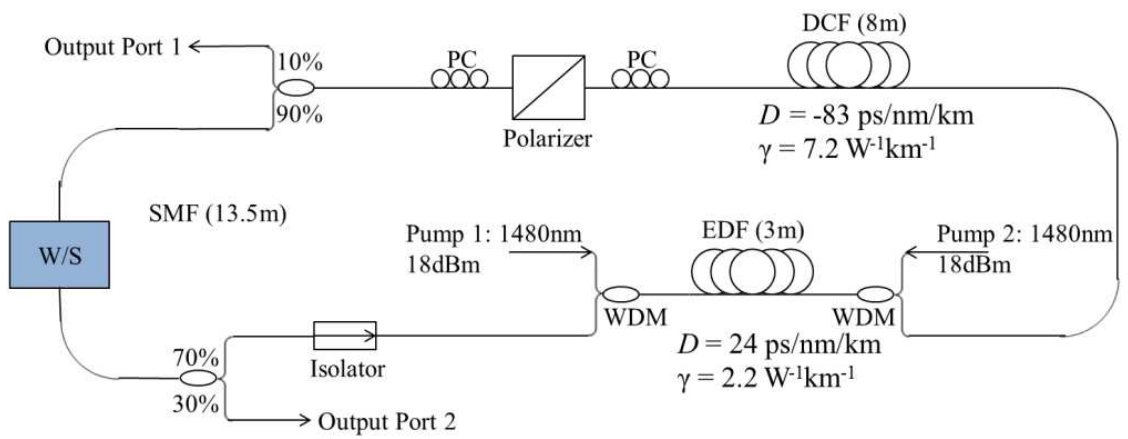

Fig. 1 Schematic of dispersion-managed passively mode-locked Er-doped fiber ring laser. W/S: Finisar Waveshaper. PC: Polarization Controller. DCF: dispersion compensating fiber. EDF: Erbium-doped fiber. WDM: Wavelength Division Multiplexing.

Fig. 1 shows a schematic of our passively mode-locked Er-doped fiber ring laser (EFRL). The mode-locking mechanism was nonlinear polarization rotation, facilitated through the inclusion of an $8 \mathrm{~m}$ long dispersion compensating fiber $(\mathrm{DCF}-D=-83 \mathrm{ps} / \mathrm{nm} / \mathrm{km}$ ) and a combination of two polarization controllers and a polarizer. The Er-doped fiber (EDF) was $3 \mathrm{~m}$ long $(D=24 \mathrm{ps} / \mathrm{nm} / \mathrm{km})$ and was pumped bi-directionally at $1480 \mathrm{~nm}$ by two 18 $\mathrm{dBm}$ pump lasers to ensure sufficient gain in the cavity. Additional single-mode fiber $(D=17 \mathrm{ps} / \mathrm{nm} / \mathrm{km})$ associated with the various components of the laser cavity amounted to a length of $13.5 \mathrm{~m}$. An isolator was used to ensure unidirectional propagation. A programmable phase and amplitude filter, the Finisar Waveshaper (W/S), was used to adaptively manage the net dispersion of the cavity. The W/S had an insertion loss of $5 \mathrm{~dB}$ and a dynamic range of $35 \mathrm{~dB}$. Under mode-locking conditions, the laser operated at a repetition rate of $6.5 \mathrm{MHz}$, which remained constant throughout the experiments described below (since there was no change in the optical length of the cavity). The W/S was programmed to have a 4-nm wide Gaussian spectral response centered at $1559 \mathrm{~nm}$ (Fig. 2(a)). In order to control the ND of the cavity we applied a range of dispersion values on the transfer function of the filter and studied the laser response. The laser pulses were observed at two points: the first was right after the normal dispersion fiber segment, while the second was at the output of W/S. 


\section{Results and Discussion}
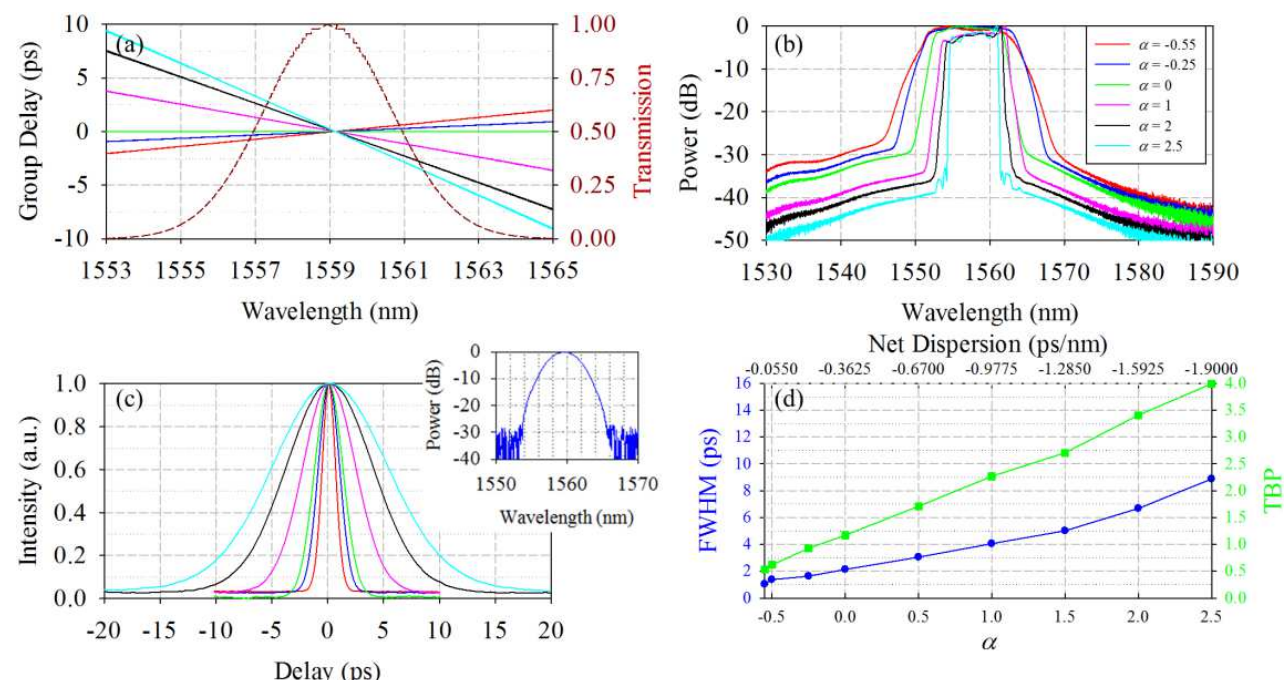

Fig. 2 (a) Transmission of the W/S and the group delay corresponding to the dispersions applied into the W.S. (b) Spectra measured at output port 1 (resolution $\mathrm{b} / \mathrm{w}=0.5 \mathrm{~nm}$ ) and (c) Autocorrelation traces measured at output port 2 for different phase profiles. The inset shows the spectrum for $\alpha=-0.55$ at output port 2. (d) FWHM and TBP of the pulses at output port 2 vs scaling factor of dispersion $(\alpha)$

In the experiment, we programmed the W/S using a range of GVD values, corresponding to the group delay profiles shown in Fig. 2(a). These values can be expressed as $\alpha D_{0}$, where $D_{0}=-0.615 \mathrm{ps} / \mathrm{nm}$, and $\alpha$ was a scaling factor of the dispersion. When there was no dispersion applied on the W/S $(\alpha=0)$, the cavity had an estimated ND of $\sim-0.363 \mathrm{ps} / \mathrm{nm}$. While nonlinearly propagating in the normal dispersion segment of the cavity the laser pulses obtained a positive chirp. When some anomalous dispersion was applied on the W/S, the ND of the cavity was reduced and this resulted in a shorter and less chirped pulse at the output of the filter. While propagating in the normal dispersion segment, pulses of a shorter width, hence a higher peak power, stimulated a larger nonlinear phase shift, in turn giving rise to a broader spectral bandwidth. Robust mode-locking could be achieved when sufficient nonlinearity had been induced and the pulse did not experience wave-breaking during its propagation in the normal dispersion segment. In the experiment, we found that as long as the value of $\alpha$ remained in the range -0.55 to $2.5(\mathrm{ND}=-0.024 \mathrm{ps} / \mathrm{nm} \sim-1.900 \mathrm{ps} / \mathrm{nm})$, robust self-starting mode-locking could be observed. From Fig. 2(b), it is clear that the spectral bandwidth of the pulses after propagation in the normal dispersion segment decreased as the absolute value of ND increased.

Figure 2(c) shows autocorrelation traces of the pulses obtained at the output of the W/S, and Fig.2(d) summarizes the pulse full-widths at half maximum (FWHM) and time-bandwidth products (TBP) indicating an almost linear increase in the TBP with the absolute value of ND. The pulses after spectral filtering had a minimum $\mathrm{TBP}$ of 0.53 (for a ND = - $0.024 \mathrm{ps} / \mathrm{nm}$ ), indicating close to transform-limited pulses.

\section{Conclusion}

In this work, we included a programmable filter in a passively mode-locked EFRL, which allowed us the capability to control the net cavity dispersion over a wide range of values, while maintaining robust pulsed operation. By simply programming a suitable dispersion profile on the filter, the pulse width could be accurately controlled between 1 and 9 ps, while the spectral broadening inside the cavity was also affected accordingly.

\section{References}

[1] R. Gumenyuk, et al., Dispersion compensation technologies for femtosecond fiber system. Applied Optics, 2011. 50(6): p. 797-801.

[2] M.A. Chernysheva, et al., Transform-limited pulse generation in normal cavity dispersion erbium doped single-walled carbon nanotubes mode-locked fiber ring laser. Optics Express, 2012. 20(21): p. 23994-24001.

[3] G. Baxter, et al., Highly programmable Wavelength Selective Switch based on Liquid Crystal on Silicon switching elements. 2006 Optical Fiber Communication Conference, 2006: p. OTuF2.

[4] D. Hillerkuss, et al., 26 Tbit s(-1) line-rate super-channel transmission utilizing all-optical fast Fourier transform processing. Nature Photonics, 2011. 5(6): p. 364-371.

[5] J. Schroder, et al., Dark and bright pulse passive mode-locked laser with in-cavity pulse-shaper. Optics Express, 2010. 18(22): p. 2271522721. 